\title{
MAGNETIC FIELD EFFECTS ON THE STRUCTURE AND EVOLUTION OF OVERDENSE RADIATIVELY COOLING JETS
}

\author{
Adriano H. Cerqueira and Elisabete M. de Gouveia Dal Pino \\ Instituto Astronômico e Geofísico, Universidade de São Paulo, \\ Av. Miguel Stéfano 4200, (04301-904) São Paulo - SP, BRAZIL \\ adriano@iagusp.usp.br, dalpino@iagusp.usp.br
}

To appear in The Astrophysical Journal

Received April; accepted July 


\begin{abstract}
We investigate the effect of magnetic fields on the propagation dynamics and morphology of overdense, radiatively cooling, supermagnetosonic jets, with the help of fully three-dimensional smooth particle magnetohydrodynamic simulations. Evaluated for a set of parameters which are mainly suitable for protostellar jets (with density ratios between the jet and the ambient medium $\eta \approx 3-10$, and ambient Mach number $M_{a} \approx 24$ ), these simulations are also compared with baseline non-magnetic and adiabatic calculations. Two initial magnetic field topologies (in $\sim$ equipartition with the gas, $\beta=p_{t h} / p_{B} \simeq 1$ ) are considered: (i) a helical field and (ii) a longitudinal field, both of which permeate both the jet and the ambient medium.

We find that, after amplification by compression and re-orientation in nonparallel shocks at the working surface, the magnetic field that is carried backward with the shocked gas into the cocoon improves the jet collimation relative to the purely hydrodynamic (HD) systems, but this effect is larger in the presence of the helical field. In both magnetic configurations, low-amplitude, approximately equally spaced $\left(\lambda \approx 2-4 R_{j}\right)$ internal shocks (which are absent in the HD systems) are produced by MHD Kelvin-Helmholtz reflection pinch modes. The longitudinal field geometry also excites non-axisymmetric helical modes which cause some beam wiggling. The strength and amount of these modes are, however, reduced (by $\sim$ twice) in the presence of radiative cooling relative to the adiabatic cases. Besides, a large density ratio, $\eta$, between the jet and the ambient medium also reduces, in general, the number of the internal shocks. As a consequence, the weakness of the induced internal shocks makes it doubtful that the magnetic pinches could produce by themselves the bright knots observed in the overdense, radiatively cooling protostellar jets.
\end{abstract}


Magnetic fields may leave also important signatures on the head morphology of the radiative cooling jets. The amplification of the nonparallel components of the magnetic fields, particularly in the helical field geometry, reduces the postshock compressibility and increases the postshock cooling length. This may lead to stabilization of the cold shell of shocked material that develops at the head against both the Rayleigh-Taylor and global thermal instabilities. As a consequence, the clumps that develop by fragmentation of the shell in the HD jets tend to be depleted in the helical field geometry. The jet immersed in the longitudinal field, on the other hand, still retains the clumps although they have their densities decreased relative to the HD counterparts. As stressed before (Cerqueira, de Gouveia Dal Pino \& Herant 1997), since the fragmented shell structure resembles the knotty pattern commonly observed in $\mathrm{HH}$ objects behind the bow shocks of protostellar jets, this result suggests that, as long as (equipartition) magnetic fields are present, they should probably be predominantly longitudinal at the head of these jets.

Subject headings: ISM: jets and outflows - MHD - stars: pre-mainsequence - stars: mass loss 


\section{Introduction}

There is increasing evidence that protostellar jets are driven by circumstellar magnetized disks associated with pre-main sequence stars (e.g. Königl \& Ruden 1993; Shu et al. 1995). Efficient mass loss in supersonic, collimated magnetized outflows is the most likely mechanism by which protostars dissipate the angular momentum accumulated during the accretion of the surrounding material. While of fundamental importance in the production and initial collimation of the jets, magnetic fields have been generally neglected in most of the analytical and numerical modeling of the structure of protostellar jets since the inferred estimates of their strength $\left(B \sim 10^{-6}-10^{-5} \mathrm{G}\right)$ suggested that they could be not dynamically important along the flow (e.g., Morse et al. 1993). However, recent observations of circularly polarized radio emission of the young stellar outflow of T Tauri S (Ray et al. 1997), for example, indicate the presence of a strong, ordered magnetic field in the flow, far away from the source, which has possibly been amplified by compression behind the shocks at the head of the outflow. After amplification and re-orientation behind the shocks, such magnetic fields may operate significant changes on the dynamics and collimation of a jet and also in its head structure as suggested by recent numerical studies (see below).

Great effort has been concentrated in the analytical and numerical study of magnetized, adiabatic, light jets, aiming mostly the investigation of extragalactic jets (see, e.g. Birkinshaw 1997 for a review). Most of that work has focused on the study of the stability properties of the beam against hydromagnetic and Kelvin-Helmholtz instabilities. In the limit of zero-velocity difference between the jet and the surrounding medium, linear theory predicts that a jet magnetically confined by a toroidal field is unstable to the pinch and kink (or helical) hydromagnetic modes (e.g., Chandrasekhar 1961) with the temporal growth rates of the pinching mode increasing with increasing density ratio, $\eta$, between the 
jet and the ambient medium. In the presence of a non-zero velocity discontinuity at the boundary layer separating the two fluids, these pure hydromagnetic modes are modified by the development of the Kelvin-Helmholtz $(\mathrm{K}-\mathrm{H})$ instability. A jet confined by a toroidal magnetic field is unstable to the fundamental and reflection pinch and kink modes of the K-H instability (Cohn 1983; Fiedler \& Jones 1984). The most unstable pinching mode in this case, at wavelengths $\lambda \sim 2 \times \pi R_{j}$ (where $R_{j}$ is the jet radius), has a destabilization length $l \propto\left(M_{j} R_{j}\right)$ (where $M_{j}=v_{j} / c_{j}$ is the jet Mach number), which is similar to the pure hydrodynamical (HD) case for jets with $\eta<\left(M_{j} / 2\right)^{3.3}$ (Cohn 1983). In the case of magnetized jets with a longitudinal magnetic field residing in an unmagnetized medium, all $\mathrm{K}-\mathrm{H}$ modes become stable for sub-Alfvénic flows (except for a small region of slow reflection modes; e.g., Bodo et al. 1989; Hardee et al. 1992; Hardee, Clarke \& Rosen 1997). In the super-Alfvénic regime, on the other hand, the growth rates of the instability are not very much different from those of pure HD flows. For example, a fundamental kink mode in the pure HD case can be identified with an Alfvén disturbance of long wavelength in the magnetohydrodynamic (MHD) case, and reflection modes at shorter wavelengths can be identified with fast magnetosonic (ms) waves reflecting off the jet boundaries. In this super-Alfvénic regime, if the jet is also supermagnetosonic $\left(M_{m s, j}=v_{j} /\left(v_{A}^{2}+c_{j}^{2}\right)^{1 / 2}>1\right.$, where $v_{A}$ is the Alfvén velocity and $c_{j}$ is the jet sound speed), then it becomes more stable with increasing $M_{m s, j}$, with the destabilization length varying approximately proportional to $M_{m s, j}\left(l \propto M_{m s, j} R_{j}\right)$ (Hardee et al. 1992). Also, strong toroidal fields of strength comparable to the poloidal field can lead to increased jet stability (Appl \& Camenzind 1992; Thiele \& Camenzind 1997).

The effects of the K-H modes on the survival of the beam, however, cannot be predicted by the linear theory alone. As in pure HD flows, the fastest growing, shortest wavelength reflection modes are expected to saturate with the formation of weak oblique shocks, while the fundamental modes may not saturate and may cause large-scale distortions and even 
disruption of the flow. Numerical simulations which can determine the end points of the operation of these instabilities confirm these predictions. Hardee et al. (1992) and Hardee, Clarke \& Rosen (1997), for example, assuming slab and cylindrical jets, respectively, with axial magnetic fields, have focused on the comparison of the scale-length of the structures generated during the nonlinear evolution and the wavelengths of maximum growth rate predicted by the linear theory. They find that the jet is not stabilized by nonlinear effects associated with increasing magnetic tension and disrupts near the resonant wavelength of the K-H kink mode. Malagoli, Bodo \& Rosner (1996) and Min (1997a, b) have extended those investigations of the nonlinear development of the $\mathrm{K}-\mathrm{H}$ instability by including diffusion and magnetic reconnection effects and found that even if the magnetic field intensity is not too large to completely suppress the $\mathrm{K}-\mathrm{H}$ instability, it is still able to mediate turbulence decay and diffusion of energy and mass across the boundary layer between jet and surrounding medium.

These studies of the stability properties of the beam against MHD K-H pinch and helical modes seem to provide potential mechanisms to explain the formation of structures such as knots and wiggles in adiabatic, supermagnetosonic light jets. Besides these stability analyses, some effort has also been spent in numerical studies of the general effects of B-fields on the global evolution and morphology of adiabatic, light jets, still in the context of extragalactic jets, assuming both passive (Clarke, Norman \& Burns 1989; Matthews \& Scheuer 1990; Hardee \& Norman 1990) and dynamical important toroidal fields (e.g., Clarke , Norman \& Burns 1986; Lind et al. 1989; Kössl, Müller \& Hillebrandt 1990). In the later case, the jet was found to be rapidly decelerated at the Mach disk with the shocked jet material being confined to a slender trans-Alfvénic plug instead of being deposited in the large cocoon observed in the purely HD light jets.

Lately, these numerical MHD studies have been extended to heavy, adiabatic jets 
(Todo et al. 1993; Hardee \& Clarke 1995; Stone, Xu \& Hardee 1997). Hardee \& Clarke and Stone et al. have focused on simulations of jets with a poloidal field propagating into an unmagnetized medium, while Todo et al. (see also Thiele \& Camenzind 1997) have considered a helical field configuration extending also to the ambient medium. Comparing the development of supermagnetosonic, adiabatic, heavy jets with axial and toroidal magnetic fields propagating into unmagnetized ambient media, Hardee \& Stone (1997) have found that the toroidal geometry suppresses the mixing and entrainment of ambient gas which is found to develop in the axial case as a consequence of the K-H instability. Also, they have found that the kink mode has a longer wavelength and smaller amplitude in the toroidal configuration.

Still almost unexplored, however, is the role played by $\mathbf{B}$-fields on the propagation dynamics and morphology of radiatively cooling, heavy jets (a scenario which is believed to occur in protostellar jets). In the limit of zero magnetic field, numerical simulations of radiatively cooling, heavy jets [e.g., Blondin, Fryxell and Königl 1990, hereafter BFK; de Gouveia Dal Pino \& Benz 1993, hereafter GB93; Stone \& Norman 1993a, 1993b, 1994; de Gouveia Dal Pino \& Benz 1994, hereafter GB94; Chernin et al. 1994; de Gouveia Dal Pino, Birkinshaw \& Benz 1996, hereafter GBB96; de Gouveia Dal Pino \& Birkinshaw 1996 (GB96)] have shown that thermal energy losses by the jet system have important effects on its dynamics. These studies have revealed that a cooling jet develops a dense, cold shell of shocked material at the head which is fragmented into clumps by Rayleigh-Taylor instability. Moreover, BFK and GB93 have found that the development of the K-H modes along the beam are inhibited by the presence of cooling. Recently, Hardee \& Stone (1997) and Stone, Xu \& Hardee (1997) (see also Massaglia et al. 1992) have examined the dynamics of $\mathrm{K}-\mathrm{H}$ unstable cooling jets. Their linear analysis indicate that the growth of the $\mathrm{K}-\mathrm{H}$ modes is very sensitive to the assumed form of the cooling function. In particular, if the cooling curve is a steep function of the temperature in the neighborhood of the equilibrium 
state, then the growth of $\mathrm{K}-\mathrm{H}$ modes is reduced relative to the adiabatic jet - a result which is consistent with previous numerical simulations (BFK; GB93). With the inclusion of a longitudinal magnetic field in a supermagnetosonic jet, Hardee \& Stone (1997) find that the magnetic field does not strongly impact the differences in the K-H stability properties between an adiabatic and a cooling HD jet, provided that the magnetic pressure does not dominate. Besides, they find that the increase in the magnetic field strength makes the linear stability properties to become more like those of an adiabatic jet. The nonlinear analysis of the growth of the $\mathrm{K}-\mathrm{H}$ modes in cooling jets in the $B=0$ limit shows similar behaviour as in the pure adiabatic cases. The jet can disrupt near the resonant frequency of the fundamental kink K-H mode, and nonlinear, higher frequency, reflection waves tend to produce low-amplitude wiggles, and can result in strong shocks in the jet beam.

In the present work, we attempt to extend these previous investigations by exploring the nonlinear effects of magnetic fields (close to equipartition with the gas) on the global evolution and morphology of radiatively cooling, heavy jets and test the morphological signatures of two different magnetic field geometries (a longitudinal and a helical configuration) on the dynamics of protostellar jets. A preliminary step in this direction was made in previous work (de Gouveia Dal Pino \& Cerqueira 1996; Cerqueira, de Gouveia Dal Pino \& Herant 1997, hereafter Paper I), where we have mainly focused on the effects of the magnetic fields in the jet head structure. With the help of three-dimensional (3-D) smooth particle magnetohydrodynamical (SPMHD) simulations, we have found that the presence of a helical magnetic field (in close equipartition with the gas) may suppress the formation of the clumpy structure that is found to develop at the head of HD jets by fragmentation of the cold shell of shocked jet material. A cooling jet immersed in a longitudinal magnetic field, on the other hand, tends to retain the clumpy morphology at its head. In the present work, we perform 3-D SPMHD simulations to address the details of the magnetic field effects over the whole structure of the radiatively cooling, heavy jets, covering a more 
extensive range of parameters, and compare with both non-magnetic and adiabatic systems.

Contemporaneously with this work, Frank et al. (1998; see also Frank et al. 1997) have performed grid-based $2 \frac{1}{2}$-D simulations of magnetized, radiatively cooling jets assuming a toroidal $\left(B_{\phi}\right)$ magnetic field and concluded that toroidal fields are able to excite the development of strong pinches along the beam. Although in our simulations we have assumed somewhat different initial conditions (see below), where both investigations overlap, the results are qualitatively similar, except for the fact that in our simulations the MHD pinch modes are found to be not strong in radiatively cooling, heavy jets. This difference, however, is mainly due to differences in the assumed initial density ratios between the jet and the ambient medium, which are much larger in our analyses (see Paper I and discussion below).

In $§ 2$, the numerical method and the initial conditions used are briefly described. In $\S 3$, we present the results of our simulations, and in $\S 4$, we address the conclusions and the possible implications of our results.

\section{Numerical Technique}

In our simulations, we consider the magnetohydrodynamics (MHD) conservation equations in the ideal approximation

$$
\begin{gathered}
\frac{d \rho}{d t}=-\rho \nabla \cdot \mathbf{v} \\
\frac{d \mathbf{v}}{d t}=-\frac{\nabla p}{\rho}+\frac{1}{4 \pi \rho}(\nabla \times \mathbf{B}) \times \mathbf{B}
\end{gathered}
$$




$$
\begin{gathered}
\frac{d u}{d t}=-\frac{p}{\rho}(\nabla \cdot \mathbf{v})-\mathcal{L} \\
\frac{d \mathbf{B}}{d t}=-\mathbf{B}(\nabla \cdot \mathbf{v})+(\mathbf{B} \cdot \nabla) \mathbf{v}
\end{gathered}
$$

where the symbols have their usual meaning (i.e., $\rho$ is the density; $\mathbf{B}$ is the magnetic field; $u$ is the specific internal energy, $\mathcal{L}$ is the radiative cooling rate, etc.). To close the system (1), an ideal equation of state is assumed:

$$
p=(\gamma-1) \rho u
$$

with $\gamma=5 / 3$.

The MHD equations above are solved (in Cartesian coordinates) using a modified version of the fully three-dimensional smooth particle hydrodynamics (SPH) code originally developed by de Gouveia Dal Pino \& Benz (1993) (see also GB94; Chernin et al. 1994; GB96; GBB96) for the investigation of the evolution of purely hydrodynamic (HD) jets.

In the SPH formalism, equations (1) are described by (e.g., Benz 1990; Stellingwerf \& Peterkin 1990; Monaghan 1992; Meglicki 1995):

$$
\begin{gathered}
\left\langle\frac{d \mathbf{v}}{d t}\right\rangle=-\sum_{j=1}^{N} m_{j}\left(\frac{p_{i}}{\rho_{i}^{2}}+\frac{p_{j}}{\rho_{j}^{2}}+\Pi_{i j}\right) \nabla_{i} W_{i j}+ \\
+\frac{1}{4 \pi \rho_{i}^{2}} \sum_{j=1}^{N}\left[m_{j}\left(\mathbf{B}_{i}-\mathbf{B}_{j}\right) \times \nabla_{i} W_{i j}\right] \times \mathbf{B}_{i} \\
\left\langle\frac{d u}{d t}\right\rangle=\frac{p_{i}}{\rho_{i}^{2}} \sum_{j=1}^{N} m_{j}\left(\mathbf{v}_{i}-\mathbf{v}_{j}\right) \nabla_{i} W_{i j}+\frac{1}{2} \sum_{j=1}^{N} m_{j} \Pi_{i j}\left(\mathbf{v}_{i}-\mathbf{v}_{j}\right) \cdot \nabla_{i} W_{i j}
\end{gathered}
$$




$$
\left\langle\frac{d B_{k}}{d t}\right\rangle=\frac{1}{\rho_{i}} \sum_{j=1}^{N} m_{j}\left(B_{i, k} \mathbf{v}_{i j}-v_{i j, k} \mathbf{B}_{i}\right) \nabla_{i} W_{i j}
$$

where $m_{j}$ is the mass of the particle $j$ (located at the position $\mathbf{r}=\mathbf{r}_{j}$ ); $\rho_{i}$ and $\rho_{j}$ are the density of the particles $i$ and $j$, respectively; $p_{i}$ and $p_{j}$ are their pressure; $\mathbf{B}_{i}$ is the magnetic field at $\mathbf{r}_{i}\left(B_{i, k}\right.$ is the $k$ component of $\left.\mathbf{B}_{i}\right) ; \mathbf{v}_{i j} \equiv \mathbf{v}_{i}-\mathbf{v}_{j}$ is the velocity difference, and $v_{i j, k}$ is $k$ component of $\mathbf{v}_{i j}$. The brackets on the left-hand side of the equations mean that the physical quantities inside them are evaluated at the position $\mathbf{r}_{i}$ (at the particle $i$ ). The term $\nabla_{i} W_{i j}$ is the gradient of the Kernel function $W_{i j}$ at the position of the particle $i$, and $\Pi_{i j}$ is the artificial viscosity which allows for appropriate treatment of shock wave dissipation. We here adopt the von Neumann-Richtmyer viscosity (e.g., Benz 1990).

The density and pressure are both calculated from the definition of discretness in SPH (e.g., Benz 1990; Monaghan 1992), and are expressed, respectively, by the equations:

$$
\begin{gathered}
\langle\rho\rangle=\sum_{j=1}^{N} m_{j} W_{i j} \\
\langle p\rangle=(\gamma-1) \sum_{j=1}^{N} m_{j} u_{j} W_{i j}
\end{gathered}
$$

As in previous work (GB93; GB94; GB96), the radiative cooling rate (due to collisional excitation and recombination) $\mathcal{L}$ in equation (1c), is implicitly calculated using the cooling function evaluated by Katz (1989) for a gas of cosmic abundances cooling from from $T \simeq 10^{6} \mathrm{~K}$ to $T \simeq 10^{4} \mathrm{~K}$. [The cooling is suppressed below $T \approx 10^{4} \mathrm{~K}$, where the transfer of ionizing radiation becomes important, and the assumption of a fully ionized flow breaks down (see, e.g., GB93; GBB96)].

The choice of a vectorial formalism to write the system of equations above (instead of a tensorial form $)$ is to ensure that the magnetic force vector $[(\nabla \times \mathbf{B}) \times \mathbf{B}]$, in each 
point of the system, is strictly perpendicular to the magnetic field vector itself. Tensorial implementations in SPMHD, like those proposed by Monaghan (1992), are subject to the development of non-physical components of the magnetic force, whose projection on the magnetic field vector has non-zero values. Furthermore, these parallel components of the magnetic force are proportional to $\nabla \cdot \mathbf{B}$ (see Brackbill \& Barnes 1980). To avoid this, we have used the vectorial form and found that those non-physical accelerations are absent in our runs [i.e., $\mathbf{F} \cdot \mathbf{B} \simeq 10^{-6}-10^{-7}$, where $\mathbf{F}=\frac{1}{4 \pi \rho}(\nabla \times \mathbf{B}) \times \mathbf{B}$ ]

There are several methods which can work well in order to maintain the divergence of the magnetic field close to the machine roundoff error in grid-based codes (e.g., Evans \& Hawley 1988; Schmidt-Voigt 1989). However, the maintenance of $\nabla \cdot \mathbf{B}=0$ in SPMHD is not an easy task. A divergence-cleaning process (e.g., Schmidt-Voigt 1989; Otmianowska-Mazur \& Chiba 1995), for example, is not applicable yet in the context of the SPMHD, and the best way we have found to diminish the effects of a potential $\nabla \cdot \mathbf{B} \neq 0$ was to avoid those non-physical accelerations by writing our system of equations in the vector formalism. Furthermore, we track the behaviour of $\nabla \cdot \mathbf{B}$ by evaluating the following quantity at each time step and position of the system (e.g, Otmianowska-Mazur \& Chiba 1995)

$$
\omega=\frac{|\nabla \cdot \mathbf{B}| \cdot h}{|\mathbf{B}|}
$$

As in Otmianowska-Mazur \& Chiba 1995 (see also Meglicki 1994, 1995 for a detailed discussion), we have set $\omega \leq 10^{-2}$ as a limit to the validity of our calculations. In general, $85 \%$ of the particles of the system keep $\omega \leq 10^{-2}$ (with almost all of them distributed around the zero value most of the time). For small periods of time, some of them may acquire an $\omega>10^{-2}$, but the scalar product between the magnetic field and the magnetic force remains very small $\left(\sim 10^{-6}-10^{-7}\right.$, in code units) over the entire evolution of the systems simulated here. 
As additional tests to check the validity of the code modified by the $\mathbf{B}$-field implementation, we have run Alfvén wave tests, similar to those suggested by Clarke (1996). In the centre of a rectangular box, a circular pulse of radius $r=\sqrt{y^{2}+z^{2}}=5 h$ (where $h$ is the smoothing length) and velocity $\mathbf{v}=v_{\text {init }} \mathbf{i}$ is set perpendicularly to the magnetic field. The latter was assumed to be in the $y$ direction in one case $\left(\mathbf{B}=B_{\text {init }} \mathbf{j}\right)$ and $\mathbf{B}=B_{\text {init }} \cdot(\mathbf{j}$

$+\mathbf{k})$ in the other case. The initial velocity was set to $v_{\text {init }} / v_{A}=\sqrt{4 \pi \rho} \cdot v_{\text {init }} / B_{\text {init }}=10^{-3}$ and $v_{\text {init }} / v_{A}=(\sqrt{2} / 2) \sqrt{4 \pi \rho} \cdot v_{\text {init }} / B_{\text {init }}=(\sqrt{2} / 2) 10^{-3}$, for each case, respectively (see Clarke 1996). Similarly to Clarke's tests, after several time steps the diffusion of the pulses was found to be strictly confined to the direction of propagation of the Alfvén waves, as depicted in Figs. 17 and 18 of Clarke (1996).

\subsection{Initial and boundary conditions}

The computational domain is represented by a 3-D rectangular box of dimensions $-17 R_{j} \leq \mathrm{x} \leq 17 R_{j},-22 R_{j} \leq \mathrm{y}, \mathrm{z} \leq 22 R_{j}$, where $R_{j}$ is the initial jet radius $\left(R_{j}\right.$ is the code distance unit). The Cartesian coordinate system has its origin at the center of the box and the jet flows through the $\mathrm{x}$-axis, and is continuously injected into the bottom of the box [at $\left.\mathbf{r}=\left(-17 R_{j}, 0,0\right)\right]$. Inside the box, the particles are initially distributed on a cubic lattice. An outflow boundary condition is assumed for the boundaries of the box. The particles are smoothed out by a spherically symmetric kernel function of width $h$, and the initial values of $h$ were chosen to be $0.4 R_{j}$ and $0.2 R_{j}$ for the ambient and jet particles, respectively.

We consider two different initial magnetic field configurations. One of them is an initially constant longitudinal B-field permeating both the jet and the ambient medium $\left[\mathbf{B}=\left(B_{x_{0}}, 0,0\right)\right]$. An observational support for this kind of configuration is suggested by the fact that some protostellar jets appear to be aligned with the main direction of the local interstellar magnetic field (e.g., Appenzeller 1989). The other adopted configuration is a 
force-free helical magnetic field which also extends to the ambient medium with a functional dependence given by (see also Todo et al. 1993):

$$
\begin{gathered}
B_{r}=0 \\
B_{\phi}(r)=B_{0}\left[\frac{0.5 A d r^{2}}{(r+0.5 d)^{3}}\right]^{1 / 2} \\
B_{x}(r)=B_{0}\left[1-\frac{A r^{2}(r+d)}{(r+0.5 d)^{3}}\right]^{1 / 2}
\end{gathered}
$$

where $r=\sqrt{y^{2}+z^{2}}$ is the radial distance from the jet axis and the (arbitrary) constants $A$ and $d$ are given by 0.99 and $3 R_{j}$, respectively. In these equations, $B_{0}$ is the maximum strength of the magnetic field and corresponds to the magnitude of the longitudinal component at the jet axis. Figure 1 displays both the longitudinal $\left(B_{x}\right)$ and the toroidal $\left(B_{\phi}\right)$ components as a function of the radial distance (in code units, for $\beta=1$ at the jet axis). The pitch angle at $1 R_{j}$ is $\approx 19^{\circ}$.

The models are parameterized by the dimensionless numbers: $i$ ) the density ratio between the jet and the ambient medium, $\left.\eta=n_{j} / n_{a} ; i i\right)$ the ambient Mach number, $M_{a}=v_{j} / c_{a}$ (where $v_{j}$ is the jet velocity and $c_{a}$ is the ambient sound speed); $i i i$ ) the jet to the ambient medium pressure ratio at the jet inlet, $\kappa=p_{j} / p_{a}$, that we assume to be equal to unit; $i v)$ the thermal to the magnetic pressure ratio, at the jet axis $\beta=p_{t h} / p_{b}$; and $v$ ) $q_{b s}=d_{\text {cool }} / R_{j}$, the ratio of the cooling length in the post-shocked gas behind the bow shock to the jet radius (see, e.g., GB93). 


\section{The Simulations}

As in previous work (e.g., GB96), based on typical conditions found in protostellar jets, we have adopted the following initial values for the parameters: $\eta=3-10$ (e.g., Morse et

al. 1992; Raga \& Noriega-Crespo 1993), an ambient number density $n_{a}=200 \mathrm{~cm}^{3}, v_{j}=398$ $\mathrm{km} \mathrm{s}^{-1}$ (e.g., Reipurth, Raga \& Heathcote 1992), $M_{a}=24$, and $R_{j}=2 \times 10^{15} \mathrm{~cm}$ (e.g., Raga 1993). In the MHD simulations, we have assumed an initial $\beta=1$ (which corresponds to a maximum initial value $B_{0}=83 \mu \mathrm{G}$ ). The subsections below present the results of the simulations we have performed for both radiatively cooling and adiabatic jets with and without magnetic fields, and Table 1 summarizes the values of the input parameters. In Table $1, M_{A_{j}}=v_{j} / v_{A_{j}}$ and $M_{m s_{j}}=v_{j} / \sqrt{v_{A_{j}}^{2}+c_{j}^{2}}$, give the initial Alfvén and magnetosonic Mach numbers, respectively. The purely hydrodynamical models are labeled with "HD" and the MHD models with "ML" and "MH" in the cases of the models with an initial longitudinal magnetic field and an initial helical field, respectively.

\subsection{Radiatively cooling jets}

Figure 2 depicts the time evolution of the magnetic field distribution of two supermagnetosonic, radiatively cooling jets: ML3r (an MHD model with initial longitudinal magnetic field configuration; top) and MH3r (an MHD model with initial helical magnetic field configuration; bottom). Both models have initial $\eta=n_{j} / n_{a}=3$ and ambient Mach number $M_{a}=24$ (see Table 1 ). We find that the field lines are amplified and reoriented across the bow shock in both magnetic field configurations. The shocked jet material which is decelerated at the head and is deposited in the cocoon carries the field lines embedded in it. Part of these lines have their polarity reversed as we can see in Fig. 2.

The cooling parameters behind the bow shock, $q_{b s} \simeq 8.0$, and the jet shock, 
$q_{j s} \simeq \eta^{-3} q_{b s} \simeq 0.3$ (see, e.g., GB93) in Fig. 2, imply that, within the head of the jet, the ambient shocked gas is almost adiabatic, whereas the shocked jet material is subject to rapid radiative cooling. The corresponding density contours and velocity field distribution maps of the models above were presented in Figs. 1 and 2 of Paper I, where they were also compared with a pure hydrodynamical model (HD3r; see also Table 1). As we have stressed in Paper I, the cold dense shell that develops at the jet head due to the cooling of the shock-heated jet material in the pure hydrodynamical case, also appears in the MHD jets. Similarly, it becomes Rayleigh-Taylor (R-T) unstable (e.g., GB93) and breaks into blobs that spill into the cocoon and show a resemblance with the Herbig-Haro objects which are observed at the head of protostellar jets. As in the HD case, the density in the shell of the MHD jets also undergoes fluctuations with time which are caused by global thermal instabilities of the radiative shock (see e.g., Gaetz, Edgar \& Chevalier 1988 and GB93 and for details). Fig. 3 shows the time evolution of the shell density $\left(n_{s h}\right)$ variations at the jet axis of the three jets, which have a period of the order of the gas cooling time $\left(t_{\text {cool }} \approx 10\right.$ years, or $t_{\text {cool }} \approx 0.3 t_{d}$, where $t_{d}=R_{j} / c_{a} \approx 38$ years corresponds to the transverse jet dynamical time). We note that although the HD and the MHD jets attain a maximum density of approximately the same magnitude at $t / t_{d} \sim 1.1$, later on the density enhancement in the MHD jets is inhibited by the presence of the $\mathbf{B}$-field, particularly in the jet with helical field (MH3r).

Fig. 2 indicates that the cold blobs that develop from shell fragmentation at the head of the MHD jets detach from the beam as they are expelled backward to the cocoon. The survival of these blobs in the cocoon seems to suffer with the presence of the magnetic fields, particularly in the helical case. Compared to the blobs of the HD jet, their density is reduced by a factor $\sim 2$ in the longitudinal case (ML3r) at the final time step $\left(t / t_{d}=1.65\right)$, and almost vanishes in the helical jet (MH3r). Fig. 4 compares the density and pressure profiles across the flow in three different positions along the MHD jets in the head region. 
The lateral blobs that develop in the cocoon, on both sides of the beam are clearly less intense in the helical case than in the longitudinal case. In the helical case, for example, we find that the toroidal (nonparallel-to-shock) component of the magnetic field, which is initially less intense than the longitudinal component (by a factor $\leq 2.5$ ), is strongly amplified by compression in the shocks at the head (by a factor $\sim 5$ ). As pointed out in Paper I, this amplification increases the cooling length behind the jet shock and reduces the density growth. As a consequence, the shell tends to stabilize against the R-T instability and the clump formation is inhibited. At the end of the evolution, the dense shell with clumpy structure which was observed to develop in the head region of the pure HD jet (Fig. 1 of Paper I) is replaced in the helical case by an elongated plug (Fig. 2) of low density material.

Fig. 2 also indicates the development of some pinching along both MHD jets. In the pure HD case, constriction occurs only very close to the jet head where the beam is over-confined by the gas pressure of the cocoon (see Figs. 1 and 2 of Paper I; see also Fig. $5 \mathrm{~b}$ below). In the MHD jet with helical field (MH3r), the toroidal component $\left(B_{\phi}\right)$, which is amplified by compression in the shocks at the head, is advected back with the shocked material to the cocoon. The associated magnetic pressure $\left(\sim B_{\phi}^{2} / 8 \pi\right)$ causes an increase in the total pressure of the cocoon relative to the pure HD case, which collimates the beam and excites the (fastest growing) small-wavelength pinch modes of the MHD K-H instability. These modes over-confine the beam and drive the approximately equally spaced internal shocks seen in the MH3r jet (Fig. 2; bottom). Along the MHD jet with longitudinal field (ML3r; Fig. 2; top), the increase in the total confining pressure of the cocoon also drives the development of the MHD K-H instabilities which excite beam pinching and internal shocks. Consistent with linear theory for K-H modes, in the supermagnetosonic regime considered here, they begin to appear at a distance $\sim M_{m s_{j}} R_{j}$, which is smaller than in the pure HD jet (see Tabel 1). 
The presence of these oblique internal shocks along the beam of the MHD jets can be testfied by the density and pressure profiles across and along the flow depicted in Fig. 5, where the pure HD model is also depicted for comparison. We see that the induced internal shocks in the MHD cases have a density contrast $n_{i s} / n_{j} \approx 4$ in the ML3r model (middle) and $n_{i s} / n_{j} \approx 5$ in the MH3r model ( or $n_{i s} / n_{s h} \simeq 0.08$ and 0.15 , respectively, where $n_{s h}$ is the density at the shell). We also note a close correlation between the pinching zones and the appearance of more intense reversed fields in the contact discontinuity between the jet and the cocoon. In both magnetic field configurations, the original longitudinal components are reoriented in the nonparallel shocks at the head and advected back to the cocoon. As a consequence, a predominantly toroidal current density distribution $\left(\mathbf{J}_{\phi}\right)$ develops around the jet. Such configuration creates a $\mathbf{J} \times \mathbf{B}$ force $\left(-J_{\phi} B_{\|}\right)$that constricts the beam triggering the MHD pinch formation.

Fig. 6 depicts the density in the midplane section of the head of three supermagnetosonic, radiatively cooling jets with $\eta=10$ after they have propagated over a distance $\sim 33 R_{j}$ : a purely HD model HD10r (top), an MHD model with initial longitudinal magnetic field configuration, ML10r (middle), and an MHD model with initial helical magnetic field configuration MH10r (bottom). Figures 7 and 8 show the time evolution of the corresponding velocity and magnetic field distributions. The other initial conditions are the same as in Fig. 2. For the time evolution depicted, there is no significant difference between the MHD jets with different $\eta$ other than those presented by their pure HD counterparts. In both HD and MHD cases, the jet with larger $\eta$ [and thus with $\left.v_{b s} \simeq v_{j} /\left(1-\eta^{-1 / 2}\right) \rightarrow v_{j}\right]$ plows into the ambient medium almost ballistically without accumulating much waste of shocked jet gas in the cocoon. The shocked ambient material is almost adiabatic $\left(q_{b s}>>1\right.$; see Table 1$)$ and accumulates in the shroud that envelops the cocoon/beam, while the shocked jet material cools much faster $\left(q_{j s}<<1\right)$ and the shock is effectively isothermal. The cold shell is thus much thinner in the larger $\eta$ jets and the head 
resembles a bullet.

As in the $\eta=3$ case, the density in the shell of the $\eta=10$ jets also undergoes variations with time (with a period of the order of the jet radiative cooling time) which are caused by the global thermal instabilities of the radiative shocks. Likewise, after reaching a similar maximum density amplitude, the MHD jets have their shell density growth inhibited due to the decrease of the shock compressibility caused by the presence of the $\mathbf{B}$-field, particularly in the jet with helical field (MH10r). We note, however, that in the HD jet, the shell density variations attain a smaller maximum density amplitude than in the MHD cases. This is possibly due to the smaller total pressure confinement that acts on its head.

In the $\eta=10$ MHD jets (ML10r and MH10r), the increase in the total confining pressure in the cocoon (due to the presence of the magnetic field) also over-confines the beam, relative to the HD jet, and drives some beam pinching.

Fig. 9a shows the density and pressure profiles across the flow in different pinch positions along the MHD and HD $\eta=10$ jets after they have propagated over a distance $33 R_{j}\left(\right.$ at $\left.t / t_{d}=1.65\right)$, which can be compared with Fig. 5 (for $\eta=3$ ). As in the $\eta=3$ models, the pinch collimation is larger in the helical MH10r jet than in the ML10r jet, while the pure hydrodynamic jet, HD10r, has not developed any internal pinches over the time scale depicted. It is interesting to note that in the MHD jets, the fastest-growing pinch modes of the K-H instability would be expected to appear only at distances $\sim M_{m s_{j}} R_{j} \sim 50 R_{j}$, which are beyond the computed scales. Thus the early development of pinches in these cases, is possibly being triggered mainly by the hydromagnetic $\theta$-pinch effect (e.g., Boyd \& Sanderson 1961; Cohn 1983). As in the $\eta=3$ case, the jet with longitudinal B-field also develops a non-axisymmetric helical (kink) mode close to the head of the jet which causes some beam twisting.

As expected, after propagating about the same distance, the pinches that develop in 
the larger $\eta$ case are less numerous, since the total amount of confining shocked material that deposits into the cocoon is comparatively smaller (see Figs. 5 and 9 for a comparison). Nonetheless, the density contrasts, $n_{i s} / n_{j}$, attained in the pinch regions of the MHD jet with longitudinal field, ML10r $\left(n_{i s} / n_{j} \approx 3-4.5\right.$, or $\left.n_{i s} / n_{s h} \sim 0.05-0.07\right)$ are approximately the same as those in the smaller $\eta$ jet (ML3r). The situation is a little more complex for the MHD jets with helical field. Over the whole jet evolution, some few pinches are found to be stronger in the larger $\eta$ jet (MH10r) (Fig. 8), but in general the density contrasts are approximately the same in both cases $\left(n_{i s} / n_{j} \lesssim 5\right.$, or $\left.n_{i s} / n_{s h} \lesssim 0.13\right)$.

\subsection{Adiabatic jets}

Fig. 10 depicts the density in the midplane section of the head of three supermagnetosonic, adiabatic jets with $\eta=3$ : a purely HD jet (HD3a, top), an MHD jet with initial longitudinal B-field, (ML3a, middle), and an MHD jet with initial helical B-field (MH3a, bottom). Figures 11 and 12 show the time evolution of the corresponding velocity and magnetic field distributions. The initial conditions are the same as in Fig. 2 (see Table 1). Previous numerical analyses comparing pure HD jets with and without radiative cooling (e.g., BFK, GB93) have shown that the presence of radiative cooling tends to reduce the strength and number of internal shocks excited by K-H instability. Consistently, the HD3a jet in Figs. 10-12 reveals the appearance of a pinching zone (at $x \simeq 1 R_{j}$ ) which is absent in its radiatively cooling counterpart (HD3r, Fig. 2; see also Figs. 1 and 2 of Paper I). Besides, the beam constriction which appears close to the head is larger in the adiabatic jet (HD3a). These results are also compatible with the predictions of the linear stability theory (Hardee \& Stone 1997), when applied in the context of the cooling function employed in this work (see $\S 1$ ). Similarly, the pinches which develop in the MHD adiabatic jets (Figs. 10-12) are generally more intense and numerous 
than in the radiatively cooling counterparts of Fig. 2. Their larger strength can be testified by direct comparison of Figs. 13 and 5 which show the transverse profiles of some pinches along the jets. In particular, for the ML3a jet, we find a chain of 8 evolving pinches, at $t / t_{d}=1.65$, against 5 in the radiatively cooling jet (ML3r), and the corresponding densities $n_{i s} / n_{j}$, are about 2 to 3 times greater than in the ML3r jet. The helical adiabatic jet MH3a displays pinches that are $40 \%$ denser than those found in the radiatively cooling jet (MH3r) at $t / t_{d}=1.65$.

We find similar results when comparing radiatively cooling and adiabatic jets with larger $\eta$. Figure 14 shows the midplane density (left) and velocity field distribution (right) for adiabatic jets with $\eta=10$ after they have propagated $\simeq 33 R_{j}$, with the same initial conditions as the cooling jets of figures 6,7 , and 8 . The corresponding magnetic field distribution is presented in Fig. 15. As before, the $\eta=10$ adiabatic jet with longitudinal B-field (ML10a) develops stronger pinches than its cooling counterpart (ML10r model) by a factor $\approx 3$. The $\eta=10$, adiabatic helical jet (MH10a), on the other hand, has pinches with densities of the same order of magnitude as those in the cooling jet (MH10r). Also, the pinches are found to be stronger in the adiabatic jets with smaller $\eta$, in both magnetic field configurations.

\section{Conclusions and Discussion}

We have investigated here the effects of magnetic fields on the structure of evolving overdense, radiatively cooling, supermagnetosonic jets with the help of 3-D SPMHD simulations and compared with purely hydrodynamical and adiabatic calculations. Two initial magnetic field configurations (with magnitude in approximate equipartition with the gas) have been examined: a longitudinal, and a helical field permeating both the jet and the ambient medium. Calculated for a set of parameters which are particularly 
appropriate to protostellar jets (with density ratios between the jet and the ambient medium $\eta \approx 3-10$, and ambient Mach number $M_{a} \approx 24$ ), our results indicate that magnetic fields have important effects on the dynamics of radiatively cooling jets. Both magnetic field geometries are able to improve jet collimation relative to the pure hydrodynamical (HD) jets, but this effect is larger in the helical field case.

As we have stressed in Paper I, the cold dense shell that develops at the jet head due to the cooling of the shock-heated jet material in the HD cases, also appears in the MHD jets. Likewise, it becomes Rayleigh-Taylor (R-T) unstable and breaks into clumps which are more visible in the smaller $\eta$ jets where the developed shell is thicker. Also as in the HD case, the shell of the MHD jets undergoes density variations with time which are caused by global thermal instabilities of the radiative shocks. However the amplification and re-orientation of the nonparallel components of the magnetic fields by the shocks at the head, particularly in the helical field geometry, reduces the postshock compressibility and increases the postshock cooling length. This tends to stabilize the shell against both the R-T and the thermal instabilities. As a consequence, the clumps that are observed to develop by fragmentation of the shell in the HD jets are depleted in the helical field geometry. The jet immersed in the longitudinal field, on the other hand, still retains the clumps although they have their densities decreased relative to the the HD counterparts. The fact that the clumpy shell structure resembles the knotty pattern of the Herbig-Haro objects which are commonly observed at the head of protostellar jets (e.g., Herbig \& Jones 1981; Brugel et al. 1985; Reipurth 1989; Heathcote et al. 1996) suggests that a longitudinal magnetic field geometry would be more likely in the outer regions of these jets than a helical field geometry (see also Paper I).

Over the computed time and length scales, internal oblique shocks along the beam are not found to develop in the HD systems examined here. On the other hand, in their MHD 
counterparts with both magnetic field configurations, the confining total pressure of the cocoon excites (the fastest growing) low-amplitude MHD K-H reflection pinching modes which drive a chain of approximately equally spaced internal shocks along the beam, but these shocks are found to be slightly stronger in the helical field case (by a factor $\approx 20 \%$ ). Also, as expected, the internal shocks tend to appear in a larger number in the smaller $\eta$ jets (due to the larger amount of confining shocked material which is deposited in the cocoon), although their densities are approximately of the same magnitude as those in the larger $\eta$ jets. A non-axisymmetric helical mode is also excited close to the head of the radiatively cooling MHD jets with longitudinal field, which causes some beam wiggling.

The number and strength of the internal shocks excited in the MHD adiabatic jets is larger than in the radiatively cooling counterparts (by a factor $\approx 2$ in both number and strength). This result is compatible with the linear stability theory (Hardee \& Stone 1997) when applied in the context of the cooling function employed in the present work, and is also compatible with previous numerical work of HD jets which has shown that the presence of radiative cooling tends to reduce the strength and number of internal shocks along the jet (e.g., BFK; GB93). Also, the pinches are found to be more numerous in the adiabatic jets with smaller $\eta$, in both magnetic field configurations.

The internal shocks are found to propagate downstream with velocities close to that of the jet head $\left(v_{b s} \approx 250 \mathrm{~km} \mathrm{~s}^{-1}\right)$. The mean distance between them $\left(\approx 2-4 R_{j}\right)$ is in agreement with the observed knots in the jets. However, the weakness of the shocks in the radiatively cooling jets $\left(n_{i s} / n_{j} \approx 3-5\right)$ makes it doubtful that they could produce by themselves the bright knots observed in protostellar jets. Probably, other mechanisms, like intermittency in the jet injection velocity, play a more relevant role in knot formation in those jets (see e.g., Raga et al. 1990; de Gouveia Dal Pino and Benz 1994; Stone and Norman 1993a). 
We should note that in the recent numerical study of magnetized radiatively cooling jets by Frank et al. (1998), they have found that toroidal fields (also in approximate equipartition with the gas) may excite the development of strong pinches along the beam. This apparent contradiction between their analysis and ours is possibly due to differences in the assumed initial conditions. Frank et al. have considered a slower and much lighter jet (with a jet Mach number $M_{j}=v_{j} / c_{s_{j}} \approx 10$ and $\eta=1.5$ ) than the cases examined here $\left(M_{j}=v_{j} / c_{s_{j}} \approx 42-76\right.$ and $\left.\eta=3-10\right)$. Thus, consistently with our results above, their smaller $\eta$ jet should be expected to produce a larger amount of more intense pinches. This result has also been confirmed by numerical simulations (not presented here) that we have performed of $\eta=1$ jets, which have produced a larger amount of pinches with slightly larger densities than the larger $\eta$ jets studied above.

Finally, we should make some remarks on the late evolution of the radiatively cooling magnetized jets. In the magnetic field maps of Figs. 2, and 8 above, we have detected the development of (sometimes strong) magnetic field reversals at the contact discontinuity between the jet and the cocoon with intensities up to 5 times their initial magnitude. As stressed in $\S 3$, field reversals occur in both investigated magnetic field configurations, because the field lines are amplified by compression in the nonparallel shocks at the jet head, and are enforced to flow backward with the shocked plasma into the cocoon. In this process, the lines are reoriented and sometimes have their polarization reversed. Beyond the integrated length and time scales depicted in the figures above, however, the increasing strength of the reversed fields due to shear at the contact discontinuity (see e.g., eq. 2c), may lead to the development of strong pinching regions which ultimately may cause jet disruption, particularly in the cases with longitudinal field. As an example, Fig. 16 depicts the time evolution of the velocity (left) and magnetic field (right) distributions of a disruption zone which occured in the late evolution of the ML3r jet of Fig. 2. We can clearly distinguish two regions with reversed B-fields whose strength increases with time, 
which are correlated with developing pinches. The inner constriction becomes so strong that it finally causes the disruption of the beam. Although shear and compression are expected to enhance $\mathbf{B}$, this amplification is possibly partially due to numerical effects. In fact, along a contact discontinuity with such a magnetic field topology with oppositely directed field lines pressed together, magnetic diffusion and reconnection may have an important role and lead to intense magnetic energy release. Of course, under the ideal-MHD approach considered here, these dissipation effects of the magnetic field have not been appropriately considered thus leading to possibly anomalous amplification of the reversed components in the late stages of the evolution of some of the jets. Further, we should note also that no jet disruption was detected in the majority of the adiabatic cases. This fact is consistent with recent numerical studies of reconnection processes in 2-D current sheets (Oreshina \& Somov 1998), which have shown that reconnection rates are smaller in adiabatic than in radiatively cooling plasmas. Although the transposition of these results to the more complex flow geometry we have investigated here is not straightforward, they seem to suggest that the appropriate consideration of magnetic field dissipation in our models will possibly decrease and even suppress the disruptive effects of the magnetic fields found in some of the radiatively cooling cases examined here in their late evolution. The transformation of magnetic energy into thermal energy of the gas will probably have important effects on the structure and emission mechanisms of the beam (see e.g., Malagoli, Bodo \& Rosner 1996; Min 1997a, b; Jones et al. 1997) and also in the process of turbulent mixing of the jet and cocoon material. Such finite magnetic resistivity effects will be addressed in a forthcoming paper. Besides, the potential signatures that magnetic fields may leave on the morphology of radiative cooling jets, especially behind the shocks, provide important constraints that can be used in future observational tests to distinguish among different candidate mechanisms for emission line production, jet collimation, and turbulent entrainment at the contact discontinuity between the jet and the cocoon. 
We are indebted to an anonymous referee for his fruitful comments. A.H.C. would like to thank the Brazilian agency FAPESP, that fully supports his work under a Ph.D. Fellowship program. E.M.G.D.P. aknowledges the Brazilian agencies FAPESP and CNPq for partial support. We are also indebted to W. Benz, M. Herant, T. Jones and J. Monaghan for useful advice and comments. The simulations were performed on a DEC 3000/600 AXP workstation, whose purchase was made possible by FAPESP. 


\section{REFERENCES}

Appenzeller, I. 1989, in Modelling the Stellar Environment: How and Why?, eds. P. Delache et al. (Gif-sur-Yvette: Editions Frontiéres), 47

Appl, S., \& Camenzind, M. 1992, A\&A, 256, 354

Benz, W. 1990, in The Numerical Modelling of Nonlinear Stellar Pulsations, ed. J.R. Buchler (Kluwer Acad. Pub., Netherlands), 269

Birkinshaw, M. 1997, in Advanced Topics on Astrophysical and Space Plasmas, ed. E.M. de Gouveia Dal Pino et al., Kluwer Acad. Publ., p. 17

Blondin, J.M., Fryxell, B.A., \& Königl, A. 1990, ApJ, 360, 370 (BFK)

Bodo, G., Rosner, R., Ferrari, A., \& Knobloch, E. 1989, ApJ, 341, 631

Boyd, T.J.M., \& Sanderson, J.J. 1969, in Plasma Dynamics (Thomas Nelson and Sons LTD)

Brackbill, J.U., \& Barnes, D.C. 1980, J. Comp. Phys., 35, 426

Brugel, E.W., Böhm, K.-H., Böhm-Vitense, E., \& Shull, J.M. 1985, ApJ, 292, L75

Cerqueira, A.H., de Gouveia Dal Pino, E.M., \& Herant, M. 1997, ApJ, 489, L185 (Paper I)

Chandrasekhar, S. 1961, in Hydrodynamic and Hydromagnetic Stability (Oxford Univ. Press)

Chernin, L., Masson, C., de Gouveia Dal Pino, E.M., \& Benz, W. 1994, ApJ, 426, 204

Clarke, D.A. 1996, ApJ, 457, 291

Clarke, D.A., Norman, M.L., \& Burns, J.O. 1986, ApJ, 311, L63 
Clarke, D.A., Norman, M.L., \& Burns, J.O. 1989, ApJ, 342, 700

Cohn, H. 1983, ApJ, 269, 500

de Gouveia Dal Pino, E.M., \& Benz, W. 1993, ApJ, 410, 686 (GB93)

de Gouveia Dal Pino, E.M., \& Benz, W. 1994, ApJ, 435, 261 (GB94)

de Gouveia Dal Pino, E.M., \& Birkinshaw, M. 1996, ApJ, 471, 832 (GB96)

de Gouveia Dal Pino, E.M., Birkinshaw, M., \& Benz, W. 1996, ApJ, 460, L111 (GBB)

de Gouveia Dal Pino, E.M., \& Cerqueira, A.H. 1996, Astro. Lett. and Communications, 34, 303

Evans, C.R., \& Hawley, J.F. 1988, ApJ, 332, 659

Fiedler, R., \& Jones, T.W. 1984, ApJ, 283, 532

Frank, A., Noriega-Crespo, A., Ryu, D., Jones, T.W. 1997 in IAU Symp. 182, Low Mass Star Formation-from Infall to Outflow, ed. F. Malbet \& A. Castets (Dordrecht: Reidel), 115

Frank, A., Ryu, D., Jones, T.W., \& Noriega-Crespo, A. 1998, ApJ (Letters), 494, L79

Gaetz, T.J., Edgar, R.J., \& Chevalier, R.A. 1988, ApJ, 239, 927

Hardee, P.E., \& Clarke, D.A. 1995, ApJ, 451, L25

Hardee, P.E., Clarke, D.A., \& Rosen, A. 1997, ApJ, 485, 533

Hardee, P.E., Cooper, M.A., Norman, M.L., \& Stone, J.M. 1992, ApJ, 399, 478

Hardee, P.E., \& Norman, M.L. 1990, ApJ, 365, 134

Hardee, P.E., \& Stone, J.M. 1997, ApJ, 483, 121 
Heathcote, S., Morse, J.A., Hartigan, P., Reipurth, B., Schwartz, R.D., Bally, J., \& Stone, J.M. 1996, AJ, 112 (3), 1141

Herbig, G.H., \& Jones, B.F. 1981, AJ, 86, 1232

Jones, T.W., Gaalaas, J.B., Ryu, D., \& Frank, A. 1997, ApJ, 482, 230

Katz, N. 1989, Ph.D. thesis, Princeton University

Königl, A., \& Ruden, S.P. 1993, in Protostars and Planets III, ed. E.H. Leavy \& J.I. Lunine (Tucson: Univ. Arizona Press), 641

Kössl, D., Müller, E., \& Hillebrandt, W. 1990, A\&A, 229, 401

Lind, K.R., Payne, D.G., Meier, D.L., \& Blandford, R.D. 1989, ApJ, 344, 89

Malagoli, A., Bodo, G., \& Rosner, R. 1996, ApJ, 456, 708

Marinho, E.P., \& Lépine, J.R.D. 1998, ApJ, (submitted)

Massaglia, S., Trussoni, E., Bodo, G., Rossi, P., \& Ferrari, A. 1992, A\&A, 260, 243

Matthews, A.P., \& Scheuer, P.A.G. 1990, MNRAS, 242, 623

Meglicki, Z. 1994, Comp. Phys. Communications, 81, 91

Meglicki, Z. 1995, Ph.D. thesis, Australian National University

Min, K.W. 1997a, MNRAS, 285, 191

Min, K.W. 1997b, ApJ, 482, 733

Monaghan, J.J. 1992, ARA\&A, 30, 543

Morse, J.A., Hartigan, P., Cecil, G., Raymond, J.C., \& Heathcote, S. 1992, ApJ, 399, 231 
Morse, J.A., Heathcote, S., Cecil, G., Hartigan, P., \& Raymond, J.C. 1993, ApJ, 410, 764

Oreshina, A.V., \& Somov, B.V. 1998, A\&A, 331, 1078

Otmianowska-Mazur, K., \& Chiba, M. 1995, A\&A, 301, 41

Raga, A.C. 1993, Ap\&SS, 208, 163

Raga, A.C., \& Noriega-Crespo, A. 1993, RMxAA, 25, 149

Raga, A.C., Binette, L., Cantó, J., \& Calvet, N. 1990, ApJ, 364, 601

Ray, T.P., Muxlow, T.W.B., Axon, D.J., Brown, A., Corcoran, D., Dyson, J., \& Mundt, R. 1997, Nature, 385, 415

Reipurth, B. 1989, A\&A, 220, 249

Reipurth, B., Raga, A.C., \& Heathcote, S. 1992, ApJ, 392, 145

Schmidt-Voigt, M. 1989, A\&A, 210, 433

Shu, F.H., Najita, J., Ostriker, E.C., \& Shang, H. 1995, ApJ, 455, L155

Stellingwerf, R.F. \& Peterkin, R.E. 1990, in Smooth Particle Magnetohydrodynamics, Technical Report, Mission Research Corporation, March 1990, MRC/ABQ-R-1248, Albuquerque, NM

Stone, J.M., \& Norman, M.L. 1993a, ApJ, 413, 198

Stone, J.M., \& Norman, M.L. 1993b, ApJ, 413, 210

Stone, J.M., \& Norman, M.L. 1994, ApJ, 420, 237

Stone, J.M., Xu, J., \& Hardee, P.E. 1997, ApJ, 483, 121 
Thiele, M., \& Camenzind, M. 1997 in IAU Symp. 182, Low Mass Star Formation-from Infall to Outflow, ed. F. Malbet \& A. Castets (Dordrecht: Reidel), 187

Todo, Y., Uchida, Y., Sato, T., \& Rosner, R. 1993, ApJ, 403, 164 


\section{TABLES}

Table 1: The models and its initial physical parameters.

\begin{tabular}{cccccccc}
\hline \hline Run & $M_{a}$ & $M_{A_{j}}$ & $M_{m s_{j}}$ & $\eta$ & $\beta$ & $q_{j s}$ & $q_{b s}$ \\
\hline HD3a & 24 & - & 41.6 & 3 & $\infty$ & - & - \\
HD10a & 24 & - & 75.9 & 10 & $\infty$ & - & - \\
ML3a & 24 & 38.1 & 28.0 & 3 & 1 & - & - \\
MH3a & 24 & 38.1 & 28.0 & 3 & 1 & - & - \\
ML10a & 24 & 70.6 & 51.4 & 10 & 1 & - & - \\
MH10a & 24 & 70.6 & 51.4 & 10 & 1 & - & - \\
\hline HD3r & 24 & - & 41.6 & 3 & $\infty$ & 0.29 & 8.03 \\
HD10r & 24 & - & 75.9 & 10 & $\infty$ & 0.02 & 16.56 \\
ML3r & 24 & 38.1 & 28.0 & 3 & 1 & 0.29 & 8.03 \\
MH3r & 24 & 38.1 & 28.0 & 3 & 1 & 0.29 & 8.03 \\
ML10r & 24 & 70.6 & 51.4 & 10 & 1 & 0.02 & 16.56 \\
MH10r & 24 & 70.6 & 51.4 & 10 & 1 & 0.02 & 16.56 \\
\hline \hline
\end{tabular}




\section{FIGURE CAPTIONS}

Figure 1: Longitudinal $\left(B_{x}\right.$; left) and toroidal $\left(B_{\phi}\right.$; right) magnetic field components (see equations 3 in the text) as a function of the radial distance $\left[r=\left(y^{2}+z^{2}\right)^{1 / 2}\right]$, for $\beta=1$ at the jet axis. The coordinates are in code units (for the magnetic fields 1 c.u. $=21.5 \mu \mathrm{G}$ ).

Figure 2: Midplane magnetic field distribution evolution of the $\eta=3$ radiatively cooling jets ML3r (top) and MH3r (bottom). The initial conditions are $\eta=n_{j} / n_{a}=3, n_{a}=200$ $\mathrm{cm}^{-3}, M_{a}=24, v_{j} \simeq 398 \mathrm{~km} \mathrm{~s}^{-1}, \beta=8 \pi p / B^{2} \simeq 1, q_{b s} \simeq 8$ and $q_{j s} \simeq 0.3$. The times and the jet head positions are: $t / t_{d}=1.40$ and $x \simeq 25 R_{j}$ (left); $t / t_{d}=1.60$ and $x \simeq 29 R_{j}$ (middle); $t / t_{d}=1.65$ and $x \simeq 30 R_{j}$ (right). Note the reorientation (and amplification), in both models, of the magnetic fields that are carried with shocked jet material into the cocoon.

Figure 3: Density of the shell at the jet axis as a function of the time for the three radiatively cooling jets presented in Fig. 2: HD3r (solid line); ML3r (dotted line) and MH3r (dashed line).

Figure 4: Density (solid line) and pressure (dashed line) across the flow at different positions along the flow in the head region of the ML3r (top) and MH3r (bottom) jets. The positions are in units of $R_{j}$. The central peak corresponds to the beam region, while the secondary peaks on both sides of the beam are the blobs which are much denser in the ML3r model (top) than in the MH3r model (bottom). These profiles are taken at $t / t_{d}=1.65$, and the density and pressure scales can be calibrated using the marker in the top region of the plots. 
Figure 5: (a) Density (solid line) and pressure (dashed line) profiles across the HD3r (top), ML3r (middle), and MH3r (bottom) jets taken in three different pinching positions along the flow (at $t / t_{d}=1.65$ ). All the profiles have been scaled as in Fig. 4. (b) The corresponding axial density along the jet axis $(y=z=0)$ showing the channel of internal shocks. The position of the three pinches depicted in (a) are labeled with arrows. Note that the origin of the coordinates along the jet axis has been shifted from $-6 R_{j}$ to 0 .

Figure 6: Gray-scale representation of the midplane density of radiative cooling jets with $\eta=10$ : a hydrodynamical jet (top; model HD10r), an MHD jet with initial longitudinal magnetic field distribution (middle; model ML10r) and an MHD jet with initial helical magnetic field distribution (bottom; model MH10r), at a time $t / t_{d}=1.65$. The initial conditions are $\eta=n_{j} / n_{a}=10, n_{a}=200 \mathrm{~cm}^{-3}, M_{a}=24, v_{j} \simeq 398 \mathrm{~km} \mathrm{~s}^{-1}, q_{b s} \simeq 16$ and $q_{j s} \simeq 0.02$. In the MHD cases, the initial $\beta \simeq 1$. The gray scale (from minimum to maximum) is given by black, light-gray, white and dark-gray. The maximum density reached by the shell at the head of the jets is $n_{s h} / n_{a} \simeq 750$ (top), $n_{s h} / n_{a} \simeq 550$ (middle) and $n_{s h} / n_{a} \simeq 450$ (bottom). (This and the remaining gray-scale plots below were built with the help of the GREY subroutine developed by Marinho \& Lépine 1998).

Figure 7: Midplane velocity field distribution evolution of the jets of Fig. 6: HD10r model (top), ML10r model (middle) and MH10r model (bottom). The times and the jet head positions are $t / t_{d}=1.40$ and $x \simeq 27 R_{j}$ (left); $t / t_{d}=1.60$ and $x \simeq 31 R_{j}$ (middle); $t / t_{d}=1.65$ and $x \simeq 33 R_{j}$ (right).

Figure 8: The same as in Fig. 7 for the magnetic field distribution of the ML10r model (top) and MH10r model (bottom). 
Figure 9: The same as in Fig. 5 for the $\eta=10$, radiatively cooling jets: HD10r (top), ML10r (middle), and MH10r jets (bottom), at $t / t_{d}=1.65$.

Figure 10: Gray-scale representation of the midplane density of adiabatic jets with $\eta=3$ : a hydrodynamical jet (top; model HD3a), an MHD jet with initial longitudinal magnetic field distribution (middle; model ML3a) and an MHD jet with initial helical magnetic field distribution (bottom; model MH3a), at a time $t / t_{d}=1.65$. The initial conditions are the same as in Fig. 6. The gray scale (from minimum to maximum) is given by black, light-gray, white and dark-gray. The maximum density reached at the head of the jets is $n_{s h} / n_{a} \simeq 37$ (top), $n_{s h} / n_{a} \simeq 39$ (middle) and $n_{s h} / n_{a} \simeq 42$ (bottom).

Figure 11: Midplane velocity field distribution evolution of the jets of Fig. 10: HD3a model (top), ML3a model (middle), and MH3a model (bottom). The times and the jet head positions are $t / t_{d}=1.40$ and $x \simeq 27 R_{j}$ (left); $t / t_{d}=1.60$ and $x \simeq 31 R_{j}$ (middle); $t / t_{d}=1.65$ and $x \simeq 33 R_{j}$ (right).

Figure 12: Midplane magnetic field distribution of the adiabatic MHD models presented in Fig. 11: ML3a (top) and MH3a (bottom).

Figure 13: (a) Density (solid line) and pressure (dashed line) profiles across the HD3a (top), ML3a (middle), and MH3r (bottom) jets taken in three different pinching positions along the flow (at $t / t_{d}=1.65$ ). The profiles have been scaled as in Figs. 4 and 5. (b) The corresponding axial density along the jet axis $(y=z=0)$, showing the channel of internal shocks. The position of the three pinches depicted in (a) are labeled with arrows.

Figure 14: Gray-scale representation of the midplane density (left) and velocity field 
distributions (right) of adiabatic jets with $\eta=10$ : a hydrodynamical jet (top; model HD10a), an MHD jet with initial longitudinal magnetic field distribution (middle; model ML10a), and an MHD jet with initial helical magnetic field distribution (bottom; model MH10a), at $t / t_{d}=1.65$. The initial conditions are the same as in Fig. 6. The gray scale (from minimum to maximum) is given by black, light-gray, white and dark-gray. The maximum density reached by the shell at the head of the jets is $n_{s h} / n_{a} \simeq 182$ (top), $n_{s h} / n_{a} \simeq 170$ (middle) and $n_{s h} / n_{a} \simeq 140$ (bottom).

Figure 15: Midplane magnetic field distribution for the jets of Fig. 14: ML10a (top), and MH10a (bottom) at $t / t_{d}=1.65$.

Figure 16: Time evolution of the velocity field (left), and the magnetic field distribution (right) of the ML3r disrupting region. The times shown, from top to bottom, are: $t / t_{d}=1.40,1.45,1.49,1.52$ and 1.55 . See the text for discussion. 
This figure "F1.gif" is available in "gif" format from: http://arxiv.org/ps/astro-ph/9808195v1 
This figure "F2.gif" is available in "gif" format from: http://arxiv.org/ps/astro-ph/9808195v1 
This figure "F3.gif" is available in "gif" format from: http://arxiv.org/ps/astro-ph/9808195v1 
This figure "F4.gif" is available in "gif" format from: http://arxiv.org/ps/astro-ph/9808195v1 
This figure "F5.gif" is available in "gif" format from: http://arxiv.org/ps/astro-ph/9808195v1 
This figure "F6.gif" is available in "gif" format from: http://arxiv.org/ps/astro-ph/9808195v1 
This figure "F7.gif" is available in "gif" format from: http://arxiv.org/ps/astro-ph/9808195v1 
This figure "F8.gif" is available in "gif" format from: http://arxiv.org/ps/astro-ph/9808195v1 
This figure "F9.gif" is available in "gif" format from: http://arxiv.org/ps/astro-ph/9808195v1 
This figure "F10.gif" is available in "gif" format from: http://arxiv.org/ps/astro-ph/9808195v1 
This figure "F11.gif" is available in "gif" format from: http://arxiv.org/ps/astro-ph/9808195v1 
This figure "F12.gif" is available in "gif" format from: http://arxiv.org/ps/astro-ph/9808195v1 
This figure "F13.gif" is available in "gif" format from: http://arxiv.org/ps/astro-ph/9808195v1 
This figure "F14.gif" is available in "gif" format from: http://arxiv.org/ps/astro-ph/9808195v1 
This figure "F15.gif" is available in "gif" format from: http://arxiv.org/ps/astro-ph/9808195v1 
This figure "F16.gif" is available in "gif" format from: http://arxiv.org/ps/astro-ph/9808195v1 